\title{
An upper limit of the number and energy of electrons accelerated at an extended coronal shock wave
}

\author{
K.-L. Klein ${ }^{1}$, R. A. Schwartz ${ }^{2}$, J. M. McTiernan ${ }^{3}$, G. Trottet ${ }^{1}$, A. Klassen ${ }^{4}$, and A. Lecacheux ${ }^{1}$ \\ ${ }^{1}$ LESIA, CNRS-UMR 8109, Observatoire de Paris, Section de Meudon, 92195 Meudon, France \\ 2 NASA Goddard Space Flight Center/SSAI Greenbelt, MD 20771, USA \\ 3 Space Sciences Laboratory, University of California Berkeley, CA 94720-7450, USA \\ 4 Astrophysikalisches Institut Potsdam, Observatorium für solare Radioastronomie, An der Sternwarte 16, 14482 Potsdam, \\ Germany
}

Received 9 April 2003 / Accepted 2 July 2003

\begin{abstract}
Type II radio emission is the most direct signature of extended shock waves in the solar corona, and a prime example of electron acceleration at a shock. Yet, the energetics of these electrons is poorly known. On 1996 August 19 and 1997 October 07 type II bursts were observed in association with flares behind the solar limb. While Earth-orbiting X-ray detectors aboard GOES and the Compton Gamma-Ray Observatory did not register emission above background, the Ulysses spacecraft, which had the candidate active region in its field of view, saw a major burst during the 1996 event. We use this peculiar configuration to infer the energetics of electrons accelerated in the low corona from the Ulysses observations, and to derive upper limits of the number and energy content of shock-accelerated electrons from the thresholds of the Earth-orbiting detectors. The inferred upper limit is similar to electron numbers estimated for large particle events at $1 \mathrm{AU}$, but the fluxes and energy fluxes of shock-accelerated electrons are much smaller than those required to produce a conspicuous hard X-ray burst.
\end{abstract}

Key words. shock waves - Sun: flares - Sun: particle emission - Sun: radio radiation - X rays: general

\section{Introduction}

Collisionless shocks are often invoked as the accelerator of high-energy particles in astrophysical plasmas. The idea is attractive because the theory of shock acceleration is relatively well understood. Furthermore, when energetic particle signatures are observed, there is often also evidence for a shock wave or a potential driver, while alternative acceleration processes, such as wave turbulence and DC electric fields, have no signature in remote sensing observations but the accelerated particles themselves. In the solar corona the clearest traces of shock waves are type II bursts at decimetric and longer radio waves (cf. reviews by Nelson \& Melrose 1985; Mann 1995; Aurass 1997). The radio emission is attributed to electrons accelerated by quasi-perpendicular (Holman \& Pesses 1983; Benz \& Thejappa 1988) or quasi-parallel shocks (Mann \& Classen 1995). The present paper uses type II bursts to address the question how many electrons are accelerated by coronal shocks and therefore how important such shocks are in particle acceleration in the solar corona.

From the drift rates of metre wave radio bursts ascribed to shock-accelerated electron beams, Mann \& Klassen (2002) derived typical energies of a few $\mathrm{keV}$. But the actual spectrum may extend beyond these values, which are imposed by the

Send offprint requests to: K.-L. Klein,

e-mail: Ludwig.Klein@obspm.fr specific wave-particle interactions that lead to observable radio waves. Bale et al. (1999) studied the source region of a type II burst from a high Mach number shock near $1 \mathrm{AU}$, and found electron beams with energies up to more than $100 \mathrm{keV}$ (Bale, pers. comm.). The most direct tracer of the energetics of electrons accelerated in the corona is hard X-ray emission. Pure type II bursts, i.e. those which are not accompanied by decimetre-metre-wave continua, were shown to occur nearly always in the decay phase or after the end of hard X-ray emission (Klein et al. 1988). It was not possible to decide from this sample if the decaying X-ray emission came from the flaring active region or whether it had also a counterpart from shockaccelerated electrons in the corona. A neat separation of the two electron populations is only possible in behind-the-limb-flares, where the thermal and non thermal X-rays from the low atmosphere are occulted, while the type II source is visible from the Earth. Two such cases, observed on 1996 August 19 and 1997 October 07, are investigated in the present paper. Radio and co-temporal X-ray observations are presented in Sect. 2. Earth orbiting satellites failed to detect any soft or hard X-ray signature from the Sun. Upper limits of numbers and energy content of shock-accelerated electrons are derived from these data in Sect. 3, and compared with fluxes or numbers of electrons inferred from other hard X-ray bursts and from particle events at $1 \mathrm{AU}$. 


\section{Radio and X-ray observations}

\subsection{Instruments}

The coronal shock waves are identified by their spectral signature observed by the Tremsdorf spectrograph of the Potsdam Astrophysical Insitute (Mann et al. 1992) in the frequency range (40-800) $\mathrm{MHz}$ and by the Nançay Decameter array (Lecacheux 2000) in the range (20-75) MHz. The Nançay Radioheliograph (henceforth NRH; Kerdraon \& Delouis 1997) mapped the Sun at five frequencies between 164 and $432 \mathrm{MHz}$, with arc-minute spatial resolution at a cadence of 2 images per second and frequency in 1996 and 1997.

$\mathrm{X}$-ray emission was observed by the Solar X-ray/CosmicRay Burst Experiment (GRB) aboard Ulysses, which viewed large parts of the anti-earthward side of the Sun during the period of interest, and by Earth-orbiting instruments: the Geostationary Operational Environmental Satellites (GOES 8 and 9), and the Burst and Transient Source Experiment (BATSE) on the Compton Gamma Ray Observatory (CGRO). The GRB hard X-ray sensor consists of two CsI(Tl) scintillators with an effective area $\sim 20 \mathrm{~cm}^{2}$ covering the energy range 20-150 keV (Hurley et al. 1992). BATSE/CGRO observes the Sun with two sets of $\mathrm{NaI}(\mathrm{Tl})$ scintillation detectors (Fishman et al. 1989): the Large Area Detectors (LADs; surface over $2000 \mathrm{~cm}^{2}$ ) operating in the nominal energy range 30-1900 keV, and the smaller Spectroscopy Detectors (SDs) in the range from $10 \mathrm{keV}$ to $110 \mathrm{MeV}$. Photon detection with GOES (Thomas et al. 1985; Kahler \& Kreplin 1991; Garcia 1994) is based on ionisation chambers with passbands $0.5-4 \AA(3.1-25 \mathrm{keV})$ and $1-8 \AA(1.5-12 \mathrm{keV})$.

\subsection{August 19}

\subsubsection{Whole Sun X-ray and radio observations}

On 1996 August 19 a type II burst occurred between 09:28 and 09:38 UT without significant accompanying radio emission at $\mathrm{dm}$ and $\mathrm{m}$-wavelengths. Its spectrum (Fig. 1, top) extended from about $330 \mathrm{MHz}$ to a few tens of MHz.

The URAP radio spectrograph (Stone et al. 1992) aboard Ulysses detected a type III burst between its high frequency limit $(0.94 \mathrm{MHz})$ and $50 \mathrm{kHz}$. The spacecraft was at ecliptic longitude $185^{\circ}$, latitude $61^{\circ}$ and heliocentric distance $4.2 \mathrm{AU}$, and viewed a large part of the backside of the Sun as seen from Earth. On account of the heliocentric distance of the spacecraft, the start of the type III burst would have been detected at $1 \mathrm{AU}$ at 9:29 $\mathrm{UT} \pm 1.5 \mathrm{~min}$ (S. Hoang, pers. comm.), similar to the metric type II burst. No type III emission was detected by the WAVES spectrograph (Bougeret et al. 1995) on the Wind spacecraft at the Lagrangian point between Sun and Earth (Waves/Wind daily spectrograms, http: / / lep694.gsfc.nasa.gov/ waves / waves.html). The WAVES and URAP observations are consistent with a type III burst originating in an active region behind the east limb of the Sun.

The count rate time histories in Fig. 1 show no significant solar X-ray emission was detected at the Earth-orbiting spacecraft (GOES, CGRO) with integration times of the order

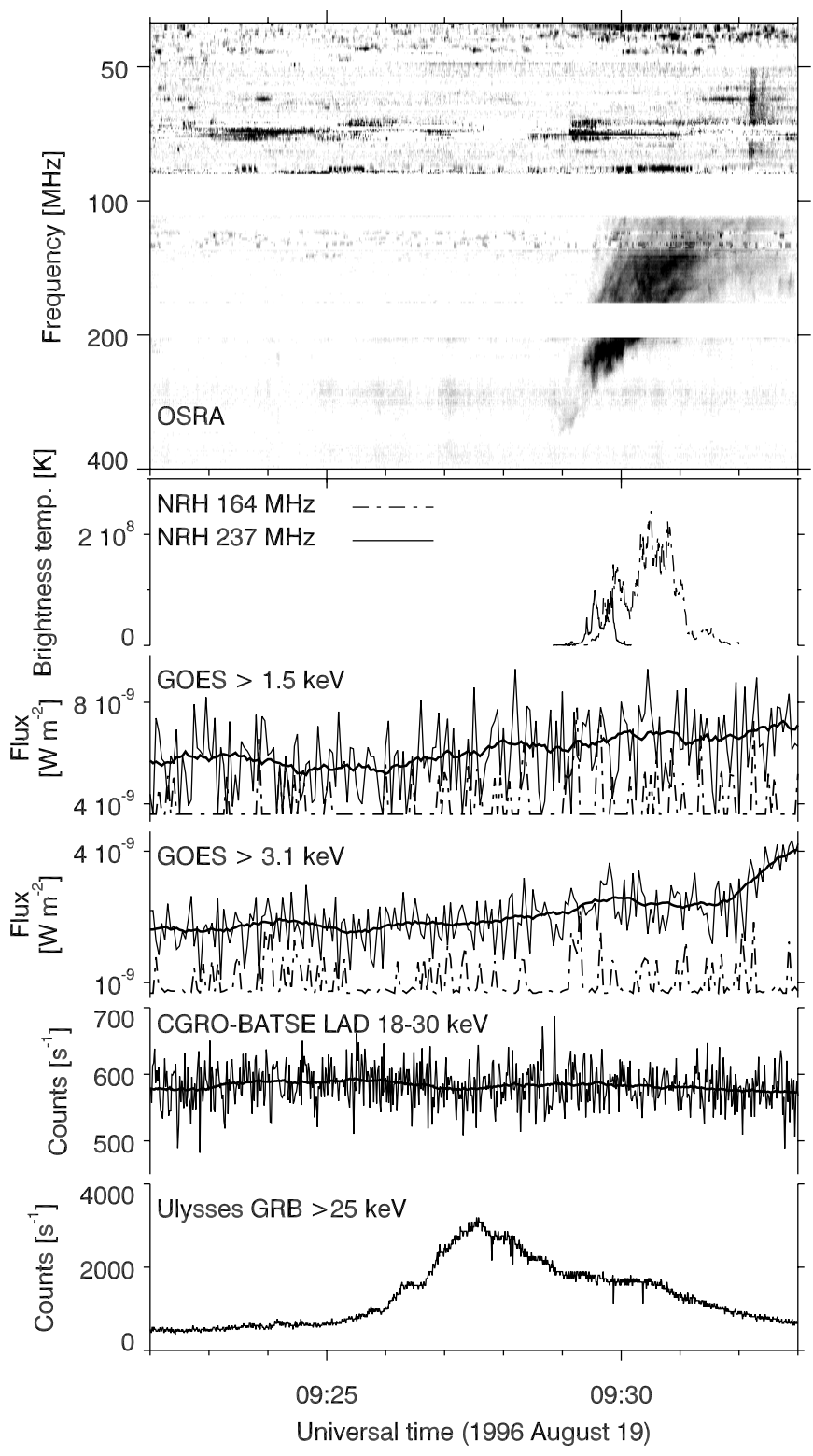

Fig. 1. Radio and X-ray emission on 1996 August 19. From top to bottom: (i) Radio spectrum of the type II event (Tremsdorf Solar Radio Observatory; dark shading means bright emission). (ii) Time histories of the radio brightness temperatures (Nançay Radioheliograph), (iii) of the GOES-8 (dashed line) and 9 (solid line) photon fluxes in the 1$8 \AA(h v>1.5 \mathrm{keV})$ and $0.5-4 \AA$ channels (thick line: running mean of the GOES-9 data over $60 \mathrm{~s}$ ), (iv) of the count rates in the $18-30 \mathrm{keV}$ photon energy range recorded by BATSE/CGRO and (v) in the 25$150 \mathrm{keV}$ range detected by GRB/Ulysses. Ulysses views a large part of the backside of the Sun as seen from Earth. The photon arrival time was corrected for the satellite position.

of $2 \mathrm{~s}$ (light jagged line) and $1 \mathrm{~min}$ (fat line). The enhancements seen by GOES 9 during and after the type II burst were not of solar origin, because they occurred at different times at GOES 8. But GRB/Ulysses observed a major hard X-ray burst. It began with weak enhancements followed by successively more intense bursts starting 9:25:15, 9:25:55, and 9:26:42 UT. The main peak occurred at 09:27:34 UT. As usual during pure type II bursts, the radio emission starts while the hard X-rays 


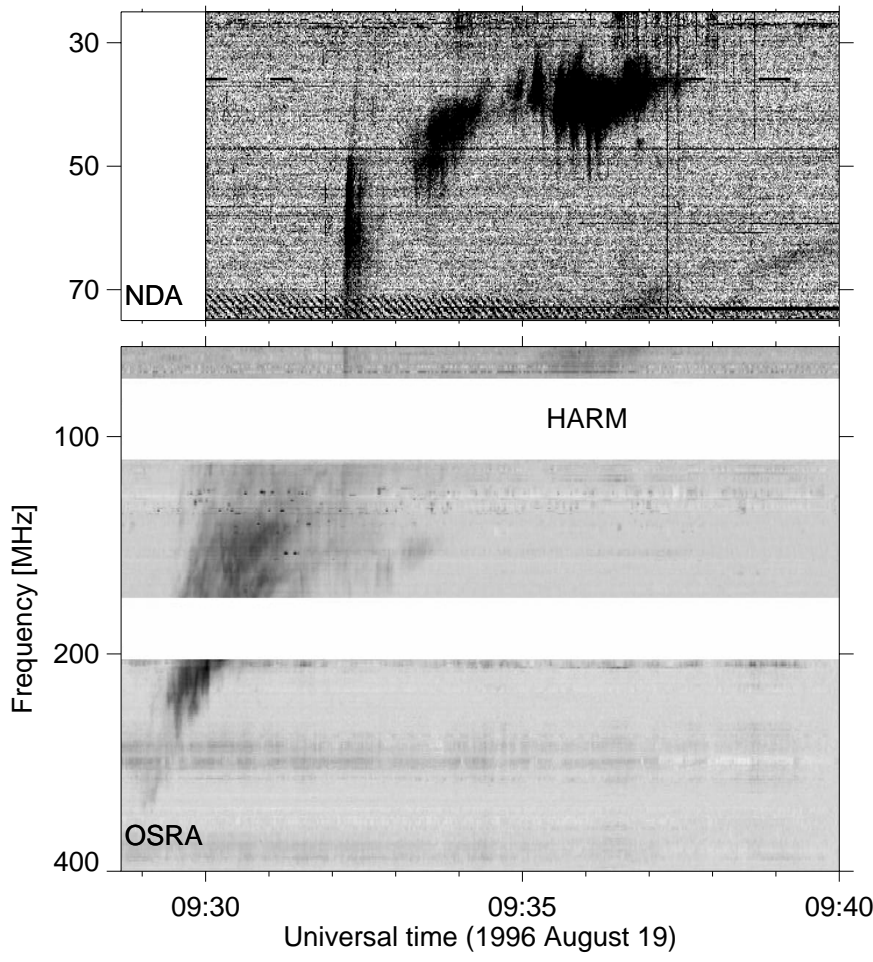

Fig. 2. Radio spectra of the type II emission on 1996 August 19 observed by the spectrographs at Tremsdorf (OSRA) and Nançay (NDA). Dark shading means bright emission. See text.

decay (Klein et al. 1988). But its start coincides with a plateau in the decay phase.

\subsubsection{Spectrum of the type II emission}

The type II spectrum is shown with more detail in Fig. 2. Contributions from the fundamental and harmonic are hard to distinguish during the phase of brightest emission. However, the weak brightening around 09:36 UT near $80 \mathrm{MHz}$, above the label "HARM" in Fig. 2, has a bright counterpart near $40 \mathrm{MHz}$, suggesting that the two are, respectively, the harmonic and the fundamental band of the type II burst. The harmonic band is continued by a weak drifting emission lane reaching $65 \mathrm{MHz}$ near 09:40 UT. From this identification we conclude that the high-frequency part of the type II burst is harmonic emission, while the low-frequency part $(<160 \mathrm{MHz})$ has contributions from the fundamental.

The fastest drifting feature of the type II spectrum is its low-frequency edge. Its slope in the time-log(frequeny) plane yields a relative drift rate $\frac{1}{v} \frac{\mathrm{d} v}{\mathrm{~d} t}=-11 \times 10^{-3} \mathrm{~s}^{-1}$, but the likely mixture of fundamental and harmonic suggests that the frequency drift inferred from a connection of the low-frequency edges across the frequency gap $200-170 \mathrm{MHz}$ is artificially high. The most significant drift is that of the bright features in the high-frequency part of the type II spectrum, at a rate $-(3.6 \pm 1.5) \times 10^{-3} \mathrm{~s}^{-1}$.

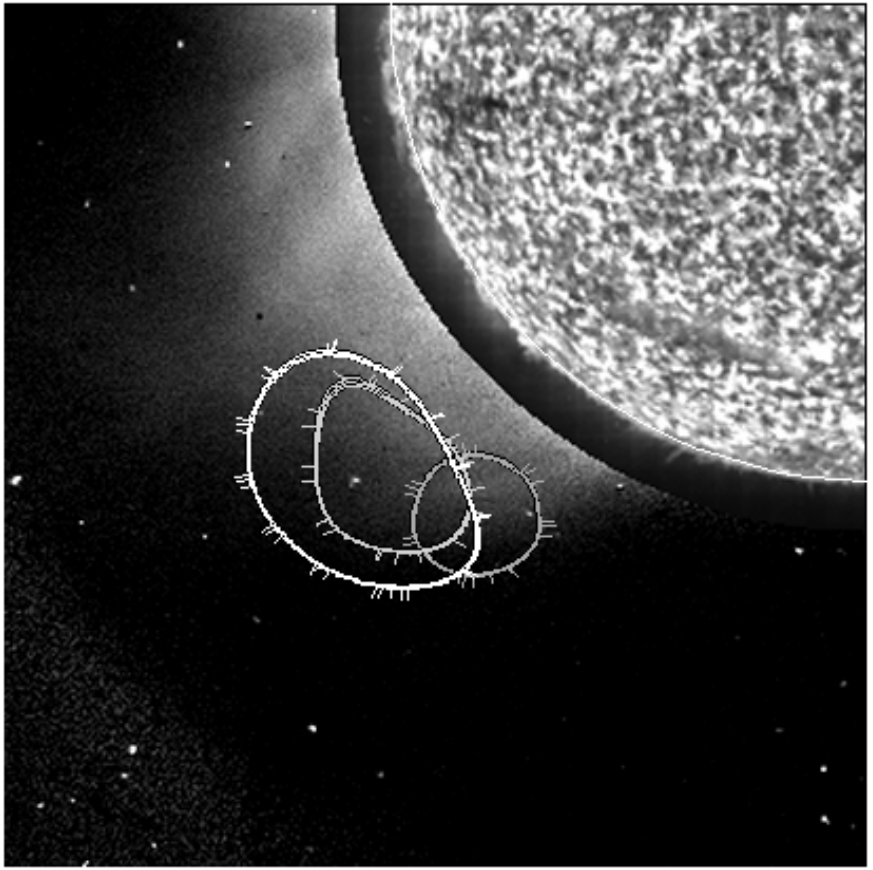

Fig. 3. Snapshot maps of the type II sources on 1996 August 19 (iso-intensity contours at half maximum; Nançay Radioheliograph, $0.5 \mathrm{~s}$ integration): $327 \mathrm{MHz}$ (09:29:01 UT), $237 \mathrm{MHz}$ (09:29:25 UT), $164 \mathrm{MHz}$ (09:30:30 UT; white contours) in the order of increasing size. Underlaid are images taken by EIT/SoHO (304 Å 08:39 UT; EIT web-page) and by LASCO/SoHO C1 (Fe XIV line, 9:26 UT; courtesy R. Schwenn).

\subsubsection{Radio imaging and identification of the parent active region}

The type II emission was observed by the NRH at 327, 237 and $164 \mathrm{MHz}$. The centroid position was stable at 327 and $237 \mathrm{MHz}$, and scattered within $\pm 0.05 R_{\odot}$ around its average value at $164 \mathrm{MHz}$. Figure 3 shows contour plots during the bright high-frequency lane identified as the harmonic band in the spectrogram. The $164 \mathrm{MHz}$ source height was only marginally above that at $237 \mathrm{MHz}$. The source heights are smaller than reported by Klein et al. (1997) because of an error in the phase calibration in that work.

The radio sources project onto a coronal streamer. The type II burst occurred in conjunction with a coronal mass ejection (cf. Klein et al. 1997) with a projected speed of $450 \mathrm{~km} \mathrm{~s}^{-1}$ (C. St.Cyr, LASCO CME list, http://lasco-www. nrl. navy.mil/lasco.html ). No active region is seen in the $304 \AA$ image in Fig. 3, and no activity was noticed in $\mathrm{H} \alpha$ on the disk (Z. Mouradian, pers. comm.). The only major active region during the period 1996 July to September (AR 7986; Biesecker et al. 1999; Démoulin et al. 2002) crossed the eastern limb on August 23. From the backward extrapolation of its positions on the disk between August 23 and 31 given in Solar Geophys. Data (weighted average of the sunspot positions) we infer that the region was located near $\mathrm{S} 12^{\circ} \mathrm{E} 137^{\circ}$ at the time of the Type II emission on August 19, corresponding to an occultation height of about $2 \times 10^{5} \mathrm{~km}$ when seen from the Earth. The backward 


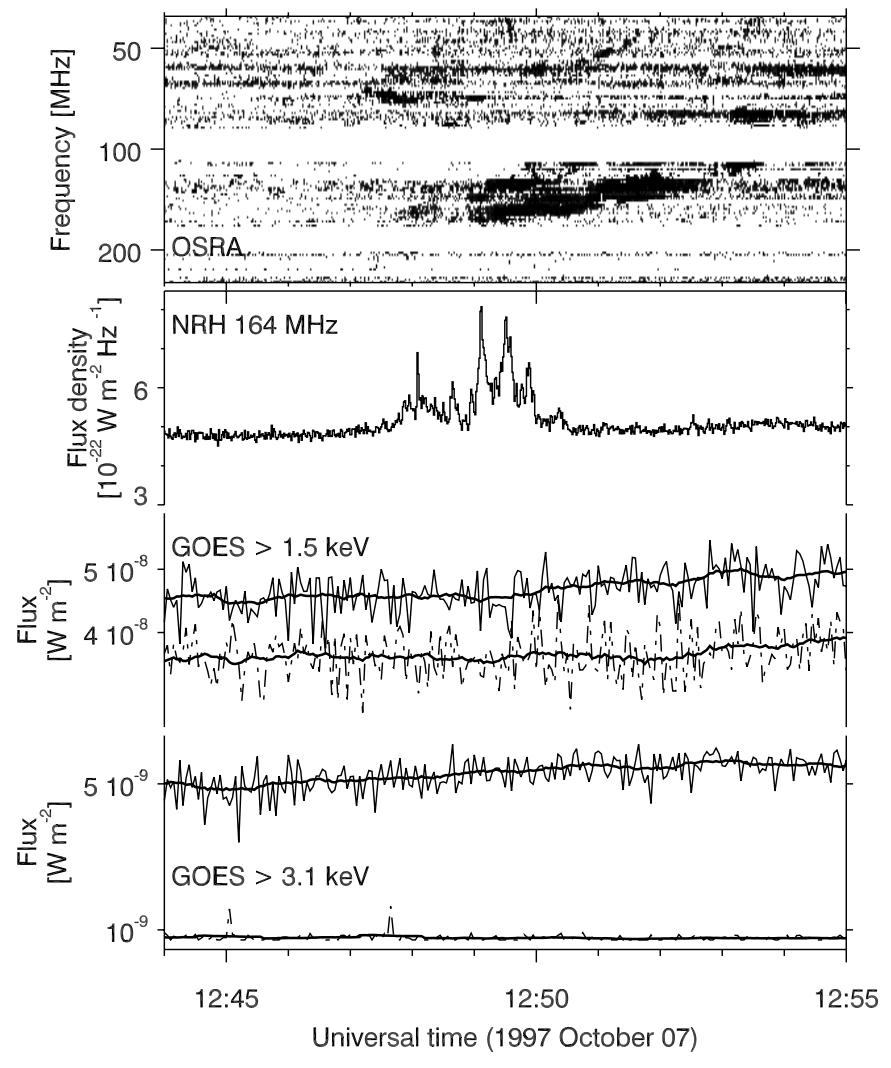

Fig. 4. Radio and X-ray emission on 1997 October 07. From top to bottom: (i) Radio spectrum of the Type II event (dark shading means bright emission). (ii) Time histories of the radio brightness temperature at $164 \mathrm{MHz}$, (iii) of the photon fluxes in the $1-8 \AA(h v>1.5 \mathrm{keV})$ and 0.5-4 A channels (thick line: running mean over 60 s).

extrapolation of the $\mathrm{H} \alpha$ flares on the disk leads to a position at E144 ${ }^{\circ}$ (occultation height $\sim 3 \times 10^{5} \mathrm{~km}$ ).

\subsection{October 07}

A similar type II burst associated with a limb-occulted flare was observed on 1997 October 07 at frequencies below $\sim 220 \mathrm{MHz}$ (Fig. 4). A slowly drifting splitted band in the range (170110) $\mathrm{MHz}$ was accompanied by weaker intermittent emission between 75 and $50 \mathrm{MHz}$, presumably traces of the fundamental band of the type II burst. The relative drift rate of the highfrequency lane was $-1.4 \times 10^{-3} \mathrm{~s}^{-1}$. The $164 \mathrm{MHz}$ source lies above the south-western solar limb (Fig. 5). The type II burst occurred at the time of a fast CME (S. Yashiro, CME catalogue at http://cdaw.gsfc.nasa.gov/CME_list/). No active region exists near the south-western limb on October 07, and no conspicuous difference is seen between the EIT images at $304 \AA$ before (07:18 UT) and after (13:19 UT) the type II burst. This argues for activity behind the south-western limb. Sunspot group NOAA 8088 crossed the limb on October 2, followed by a spotless plage region $32^{\circ}$ east of it (named AR 8087 until September 26, when it was identified by its sunspots for the last time). The extrapolation of the positions on the disk (September-October 1997) leads to S29 ${ }^{\circ} \mathrm{W} 152^{\circ}$ at $13 \mathrm{UT}$ on October 07 for AR 8088, and S26 ${ }^{\circ} \mathrm{W} 120^{\circ}$ for AR 8087, corresponding to an occultation height of $(0.8-3) \times 10^{5} \mathrm{~km}$.

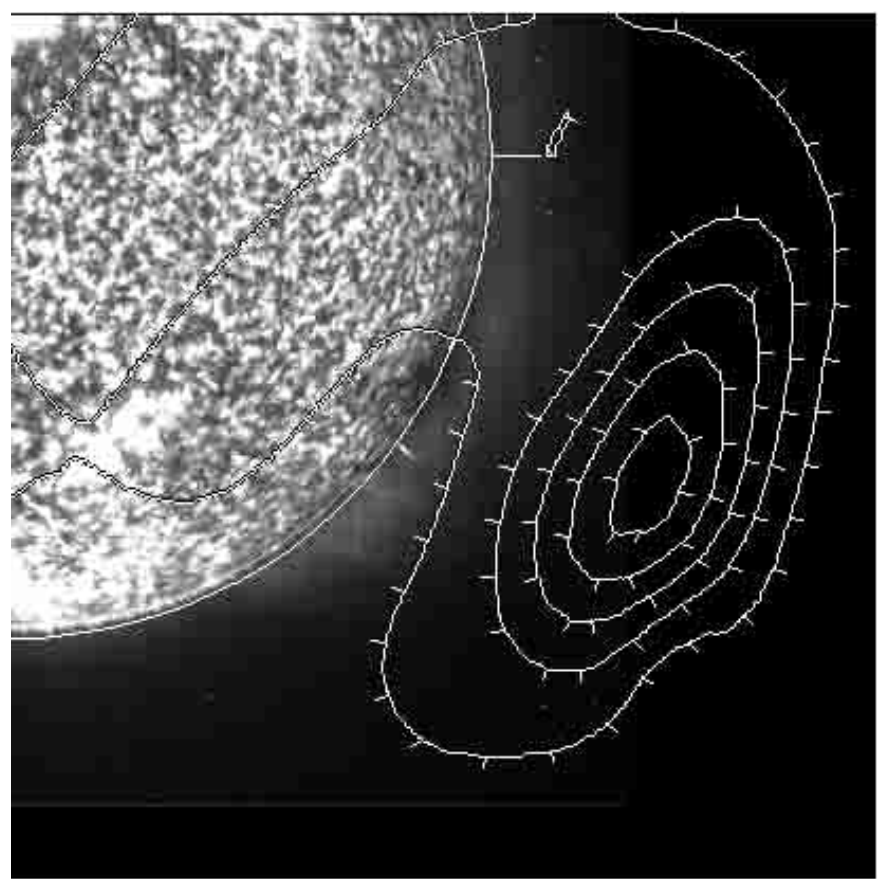

Fig. 5. Snapshot map of the type II radio source at $164 \mathrm{MHz}$ on 1997 October 07 12:49:30 UT (1 s integration time; contours from 10\% to $90 \%$ of maximum brightness) superposed on the image at $304 \AA$ taken at 13:19 UT.

The WAVES/Wind radio spectrograph detected a bright type III burst at frequencies below $5 \mathrm{MHz}$ during the metric type II emission, and several others at successively lower frequencies in the following $30 \mathrm{~min}$. URAP/Ulysses saw the same burst group about $35 \mathrm{~min}$ later at heliocentric distance 5.3 AU. This kind of type III bursts had been termed "shockaccelerated", "shock-associated" (Cane et al. 1981; Dulk et al. 2000) or "III l" (Cane et al. 2002), and identified as a typical counterpart of solar energetic particle events by the latter authors. A particle event at proton energies above $20 \mathrm{MeV}$ was indeed reported by Cane et al. (2002) on October 07. The release of protons and electrons started more than $20 \mathrm{~min}$ after the metric type II burst (A. Posner, S. Krucker, pers. comm.).

The GOES see mainly a quiet X-ray background (Fig. 4) at levels comparable with 1996 August 19 in the high-energy channel, and higher at the low energies, due to the rising activity cycle. A slow rise exceeding the general level of fluctuations appears in the low-energy channel of both spacecraft after 12:51 UT, but no enhancement is seen that correlates with the type II radio emission. BATSE/CGRO was in the Earth's shadow between 12:38 and 13:14 UT. No event was seen by GRB/Ulysses, although the satellite had both candidate active regions in its field of view.

\subsection{Summary of observations}

The type II bursts of 1996 August 19 and 1997 October 07 were probably associated with flares on the anti-earthward solar hemisphere, with occultation heights $>2 \times 10^{5} \mathrm{~km}$ and $>0.8 \times 10^{5} \mathrm{~km}$, respectively. The electrons emitting the type II bursts produced no detectable X-ray emission at photon 
energies above $2 \mathrm{keV}$. We shall use this non-detection in the following section to examine the energetics of the shockaccelerated electrons. Because of the more complete data set, we focus on the 1996 August 19 event.

The reasoning is justified provided the radio source detected from Earth is indeed the region where the radio emission is generated by shock-accelerated electrons. An alternative is that the actual site where radio waves were emitted was behind the limb, and that the waves were guided by dense fibres to the apparent source seen by the NRH. This argument has been extensively used to discuss the positions of fundamental type II sources (Duncan 1979; Benz 1993, ch. 11.5), and does not apply to the harmonic. Evidence that propagation effects do not significantly alter the position of a harmonic type II radio source comes from the observation of a type II burst with bands at the fundamental, the harmonic and the second harmonic of the plasma frequency (Zlotnik et al. 1998). In this case the first and second harmonic source were cospatial. Since the influence of coronal propagation must decrease with increasing harmonic number, the finding suggests that propagation effects do not shift the centroid position of a harmonic type II burst in a measurable way.

\section{Analysis of the 1996 August 19 event}

\subsection{Estimation of the shock speed}

The speed of the coronal shock wave can be estimated independently from the spectrum and from the source location.

The first estimate of the exciter speed is the distance from the active region (presumably at $S 12^{\circ} \mathrm{E} 137^{\circ}$ ) to the site of the type II emission at $237 \mathrm{MHz}$ divided by the travel time. If the radio source is located in the plane of the sky, its heliocentric distance is $1.4 R_{\odot}$, and the distance from the active region, $8.2 \times$ $10^{5} \mathrm{~km}$. The distance from the active region has a minimum value of $6.5 \times 10^{5} \mathrm{~km}$ if the type II source is at a heliocentric distance of $1.6 R_{\odot}$, at $\mathrm{S} 43^{\circ} \mathrm{E} 121^{\circ}$. If the exciter of the shock wave is launched in the active region at the start of the earliest hard X-ray signatures (09:23:17 UT), the time delay of $358 \mathrm{~s}$ to the onset of the type II burst implies an average speed between 1800 and $2300 \mathrm{~km} \mathrm{~s}^{-1}$.

The second estimate exploits the frequency drift. If the type II source is envisioned as the region where a dense coronal structure, e.g. a streamer, is intersected by a shock wave launched in a remote active region (e.g., Stewart 1984), the exciter speed of the type II burst is related to the frequency drift by $v \cos \alpha=\frac{2 H}{v} \frac{\mathrm{d} v}{\mathrm{~d} t}$, where $H$ is the density scale height and $\alpha$ the angle between the shock normal and the density gradient. The drift rates of the brightest features in the high-frequency band of the type II burst (Sect. 2.2) yield speeds in the range $v \cos \alpha=(720 \pm 300) \frac{H}{10^{5} \mathrm{~km}} \mathrm{~km} \mathrm{~s}^{-1}$. The barometric scale height for a plasma of $2 \times 10^{6} \mathrm{~K}$ at heliocentric distance $1.4 R_{\odot}$ is about $2 \times 10^{5} \mathrm{~km}$. $H$ is independent of the coronal structure (Koutchmy 1994). We conclude that despite the ambiguity of both speed determinations, a shock speed near $2000 \mathrm{~km} \mathrm{~s}^{-1}$ is consistent with the spectrum as well as with the position and timing of the type II source.
Table 1. X-ray photon fluxes $K$ at $50 \mathrm{keV}$ (single power law).

\begin{tabular}{lllcc}
\hline \hline Detector & Energy & \multicolumn{3}{c}{$3 \sigma$ upper limit of photon flux } \\
& {$[\mathrm{keV}]$} & $\gamma=3$ & 4 & 5 \\
\hline GOES 1-8 $\AA$ & $\geq 1.5$ & $1 \times 10^{-2}$ & $8 \times 10^{-4}$ & $5 \times 10^{-5}$ \\
GOES 0.5-4 $\AA$ & $\geq 3.1$ & $6 \times 10^{-3}$ & $7 \times 10^{-4}$ & $6 \times 10^{-5}$ \\
BATSE SD & $10-18$ & $6.6 \times 10^{-3}$ & $2.0 \times 10^{-3}$ & $5.6 \times 10^{-4}$ \\
BATSE LAD & $18-30$ & $2.7 \times 10^{-3}$ & $1.5 \times 10^{-3}$ & $8.0 \times 10^{-4}$ \\
BATSE LAD & $30-60$ & $2.2 \times 10^{-3}$ & $1.7 \times 10^{-3}$ & $1.3 \times 10^{-3}$ \\
\hline Ulysses GRB & $25-150$ & \multicolumn{3}{c}{ Peak $(9: 27: 21): \gamma=4.8, K=4.3$} \\
& \multicolumn{4}{c}{ Decay $(9: 30: 01): \gamma=5.3, K=1.2$} \\
\hline
\end{tabular}

\subsection{Derivation of electron spectra from the $X$-ray observations}

We suppose the photon spectrum incident on the X-ray detectors is a power-law as a function of photon energy $\epsilon$

$\Phi(\epsilon)=K \cdot\left(\frac{\epsilon_{1}}{\epsilon}\right)^{\gamma}$ [photons $\left.\mathrm{keV}^{-1} \mathrm{~cm}^{-2} \mathrm{~s}^{-1}\right]$,

where $\epsilon_{1}=50 \mathrm{keV}$ is a reference energy.

The GRB count rates are binned into intervals of $16 \mathrm{~s}$ and 16 detector channels in the range $25-150 \mathrm{keV}$. The photon flux at $50 \mathrm{keV}(K)$ and the spectral index at the peak of the event and during the plateau phase of the decay are given in Table 1 . We suppose that these hard X-rays are thick-target bremsstrahlung from a power-law injection spectrum of electrons, and compute the flux and the energy flux of electrons above an assumed lowenergy cutoff $E_{0}$ using the formulae of Hudson et al. (1978, see Appendix). The results for the peak of the X-ray event $(\gamma=4.8)$ and in the plateau phase during its decay $(\gamma=5.3)$ are listed in Table 2. The total energy content of electrons above $20 \mathrm{keV}$ $(25 \mathrm{keV})$ producing the entire hard $\mathrm{X}$-ray burst is $7 \times 10^{31}(3 \times$ $10^{31}$ ) ergs.

From the non-detection of the X-ray event by BATSE and GOES we can infer upper limits of the photon flux emitted by the shock-accelerated electrons upstream of the type II shock. To this end power-law photon spectra with different indices are convolved with the response functions of the detectors to predict count rates (BATSE) or energy fluxes (GOES). For each spectral index the maximum amplitude of the photon spectrum is determined so that the predicted count rate is equal to the $3 \sigma$ level in the BATSE data (for an integration time of $2.048 \mathrm{~s}$ ) or that the predicted energy flux does not produce any excess in the GOES-8 measurements (09:24-09:32 UT) that could be identified by visual inspection. The upper limits inferred from the various data sets of GOES and BATSE are listed in Table 1 for spectral indices 3, 4, 5. Depending on the spectral index, the ratio of the $3 \sigma$-upper limit of the photon fluxes at $100 \mathrm{keV}$ seen by BATSE to photon fluxes seen by Ulysses (both normalised to $1 \mathrm{AU})$ is in the range $(0.3-2) \times 10^{-3}$ for the main peak, $(1-9) \times 10^{-3}$ for the plateau in the decay phase.

$\mathrm{X}$-ray bremsstrahlung from the plasma ahead of a coronal shock wave is produced in a thin target, where the energetic electrons escape from the source before losing a significant amount of energy through collisions. Under these 
Table 2. 1996 August 19: accelerated electrons.

\begin{tabular}{|c|c|c|c|c|c|c|c|c|c|c|}
\hline \multirow[b]{2}{*}{$\gamma$} & \multicolumn{10}{|c|}{ Cutoff energy $E_{0}[\mathrm{keV}]$} \\
\hline & 2 & 5 & 10 & 20 & 25 & 2 & 5 & 10 & 20 & 25 \\
\hline
\end{tabular}

Type II source: thin target model (BATSE/CGRO, GOES)

\begin{tabular}{|c|c|c|c|c|c|c|c|c|c|c|}
\hline \multirow[b]{2}{*}{3} & \multicolumn{5}{|c|}{ Maximum number of electons $E>E_{0}$} & \multicolumn{5}{|c|}{ Maximum energy, $E>E_{0}[\mathrm{erg}]$} \\
\hline & $3 \times 10^{36}$ & $4 \times 10^{35}$ & $2 \times 10^{35}$ & $2 \times 10^{34}$ & $1 \times 10^{34}$ & $3 \times 10^{28}$ & $1 \times \overline{10^{28}}$ & $8 \times 10^{27}$ & $2 \times 10^{27}$ & $2 \times 10^{27}$ \\
\hline 4 & $6 \times 10^{36}$ & $5 \times 10^{35}$ & $3 \times 10^{35}$ & $4 \times 10^{34}$ & $2 \times 10^{34}$ & $3 \times 10^{28}$ & $7 \times 10^{27}$ & $7 \times 10^{27}$ & $2 \times 10^{27}$ & $2 \times 10^{27}$ \\
\hline 5 & $1 \times 10^{37}$ & $5 \times 10^{35}$ & $4 \times 10^{35}$ & $5 \times 10^{34}$ & $4 \times 10^{34}$ & $5 \times 10^{28}$ & $6 \times 10^{27}$ & $9 \times 10^{27}$ & $2 \times 10^{27}$ & $2 \times 10^{27}$ \\
\hline \multicolumn{11}{|c|}{ Low atmosphere: thick target model (GRB/Ulysses) } \\
\hline & \multicolumn{5}{|c|}{$\underline{\text { Flux of electons } E>E_{0}}\left[\mathrm{~s}^{-1}\right]$} & \multicolumn{5}{|c|}{ Energy flux, $E>E_{0}\left[\mathrm{erg} \mathrm{s}^{-1}\right]$} \\
\hline 4.8 & - & - & - & $7.3 \times 10^{36}$ & $2.4 \times 10^{36}$ & - & - & - & $2.9 \times 10^{29}$ & $1.2 \times 10^{29}$ \\
\hline 5.3 & - & - & - & $3.9 \times 10^{36}$ & $1.2 \times 10^{36}$ & - & - & - & $1.5 \cdot 10^{29}$ & $5.8 \times 10^{28}$ \\
\hline
\end{tabular}

conditions the instantaneous number of electrons above energy $E_{0}$ can be inferrred from the photon spectrum (cf. Appendix, after Hudson et al. 1978). It is proportional to the ambient proton density, which is constrained by the observation of type II radio emission at the harmonic of the electron plasma frequency. This implies $n_{0}=1.7 \times 10^{8} \mathrm{~cm}^{-3}$ at $237 \mathrm{MHz}$. Table 2 gives the upper limits of the instantaneous number and energy content of the shock-accelerated electrons for the assumed power law photon spectra. The values were computed using for each photon spectral index the maximum photon flux derived from the detection limits in Table 1 of GOES (1-8) $\AA$ $\left(E_{0}=2 \mathrm{keV}\right)$ and $(0.5-4) \AA\left(E_{0}=5 \mathrm{keV}\right)$, BATSE SD $\left(E_{0}=10 \mathrm{keV}\right)$, BATSE LAD $18-30 \mathrm{keV}\left(E_{0}=20 \mathrm{keV}\right)$ and $30-60 \mathrm{keV}\left(E_{0}=25 \mathrm{keV}\right)$. The values of $E_{0}$ are chosen for comparison with published work. Since the duration of the type II emission implies that electrons are accelerated during a time interval of about $1 \mathrm{~min}$, the upper limits in Tables 1 and 2 are reduced by factors $\sqrt{30} \simeq 5.5$ for BATSE and $\sqrt{20} \simeq 4.5$ for GOES by integration, as illustrated by the smoothed count rates and energy fluxes plotted as fat lines in Fig. 1.

\subsection{Comparison with electrons accelerated during hard $X$-ray bursts and solar energetic particle events}

Measured energy fluxes of hard X-ray emitting electrons above $20 \mathrm{keV}$ range from $\sim 10^{24} \mathrm{erg} \mathrm{s}^{-1}$ in microflares (Lin et al. 1984) to $\sim 10^{32} \mathrm{erg} \mathrm{s}^{-1}$ in giant flares (Kane et al. 1995). In a survey of about 12000 hard X-ray bursts observed with HXRBS/SMM, Crosby et al. (1993) found that the frequency distribution of peak energy fluxes above $25 \mathrm{keV}$ follows a power law above $10^{27} \mathrm{erg} \mathrm{s}^{-1}$, and is flat below, probably due to the detector threshold. Although the thick target formula employed by Crosby et al. gives a 1.8 times higher value than the one used in Table 2, the peak energy flux detected by Ulysses on 1996 August 19 is within the highest decade of the HXRBS statistics.

For the sake of comparison with the HXRBS values, we transform the upper limit of the instantaneous energy content of the shock-accelerated electrons (Table 2) into energy flux assuming that the typical acceleration time of the electrons by the coronal shock is of the order of $1 \mathrm{~s}-\mathrm{a}$ value which lies in between the duration of radio signatures of shock-accelerated electron beams in spectra with high time resolution ("herringbone structures", $0.3 \mathrm{~s}$ : Slottje 1981; Zlotnik et al. 1998) and their repetition rate in moderately resolved spectra $(3 \mathrm{~s}$; Cairns \& Robinson 1987). It also corresponds to a typical time of flight of an electron with speed c/3 through the type II source, which has a size of order $10^{5} \mathrm{~km}$. The maximum instantaneous energy flux of electrons above $25 \mathrm{keV}$ compatible with the BATSE limit during the type II burst is then of the order of $10^{27} \mathrm{erg} \mathrm{s}^{-1}$ (cf. Table 2) for $2 \mathrm{~s}$ integration time, and of some $10^{26} \mathrm{erg} \mathrm{s}^{-1}$ for $1 \mathrm{~min}$. integration. If those electrons had been injected into a thick target, they would have emitted a weak HXRBS event (see Crosby et al. 1993, Fig. 5). One to two orders of magnitude more electrons than the type II shock can at most provide are required for the moving coronal hard X-ray source analysed by Hudson et al. (2001) and for the coronal X-ray emission, at heights $\sim 2 \times 10^{5} \mathrm{~km}$, of electrons above $2 \mathrm{keV}$ during the large particle event of 1984 February 16 (Kane et al. 1992).

The number of energetic electrons released into the interplanetary space during transient solar activity ranges from $\sim 3 \times 10^{35}$ electrons above $2 \mathrm{keV}$ (Jackson \& Leblanc 1991, their Table 1) in the numerous impulsive low energy beams (2-15 keV, cf. Lin 1985; Dulk et al. 1998) to $10^{33}$ to $10^{35}$ electrons with energies above $20 \mathrm{keV}$ during large events (Lin et al. 1982). The numbers during large events bracket the upper limits of shock-accelerated electrons inferred from our $\mathrm{X}$-ray analysis. The major uncertainty of the number estimation from the particle measurements is the volume into which the electrons are injected.

\section{Discussion}

The type II bursts of 1996 August 19 and 1997 October 07 occurred in configurations where the presumable parent active region was hidden to Earth-orbiting detectors. GOES saw no excess above background at photon energies $\geq 1.5 \mathrm{keV}$ during either event, and BATSE, which observed the Sun only during 
the 1996 flare, saw none above $10 \mathrm{keV}$. During the same event Ulysses/GRB observed a huge burst which hence was emitted at heights $\leq(2-3) \times 10^{5} \mathrm{~km}$ above the photosphere, the height range that was occulted as seen from Earth.

Since type II bursts from shock-accelerated electrons were the only radio emission observed at the Earth during the two events, these flares provided a neat distinction between the radiative signatures of electrons accelerated in the active region and at the coronal shock. Provided the observed type II source is indeed the region where shock-accelerated electrons radiate, and not the mirror image of a source behind the limb, electrons accelerated by the type II shock were not numerous enough to produce a detectable X-ray signature at photon energies above $1.5 \mathrm{keV}$. The high sensitivity of the BATSE detectors and the complete occultation of the thermal X-ray emission from the flare in the GOES data provide a unique opportunity to infer quantitative constraints on shock-accelerated electrons. The coronal type II shock is a less efficient accelerator than the acceleration processes which produce the electrons radiating the hard X-ray emission during the 1996 August 19 event or other hard X-ray bursts.

Although this evaluation is founded only on one specific event, the fact that type II bursts without metric continuum are in general not accompanied by hard X-ray emission from the low corona (Klein et al. 1988; Klassen et al. 2002) suggests that it has general validity at least for electrons above $20 \mathrm{keV}$. When hard X-rays, radio continua and type II bursts occur together, the emitting electrons must be accelerated at lower heights. The 1996 August event provides a specific illustration: while the occurrence of a plateau in the decaying hard X-ray emission at the time of the type II burst could be an argument that the shock wave accelerates the X-ray emitting electrons, along the lines of reasoning in Frost \& Dennis (1971), the estimates of numbers and energies in Table 2 clearly refute this idea. This conclusion confirms and completes earlier objections against the central role of the type II shock in the acceleration of interacting electrons during long duration flares, which emphasised that (i) the lifetime of the energetic electrons required time-extended acceleration in the corona which was incompatible with a travelling agent such as a single shock (Klein et al. 1983), (ii) the X-ray emitting electrons were confined in much smaller structures than the coronal shock (Kahler 1984), (iii) gradual hard X-ray bursts may occur without a type II burst (Cliver et al. 1986).

Transient enhancements of the electron flux in interplanetary space are not inconsistent with the upper limits for the numbers and energy of shock-accelerated electrons, but the numbers of electrons required in large electron events, i.e. those where shock acceleration in the corona is most often advocated (Lin et al. 1982; Mann et al. 1999; Klassen et al. 2002; Simnett et al. 2002), are close to or sometimes even larger than the upper limits allowed by the present analysis. The present analysis shows that shock-acceleration need not be the most plausible mechanism at work, even when fast ejecta exist. Furthermore, energetic electron events are nearly always accompanied by electron acceleration in the flaring active region or its vicinity. As an example, the electron event associated with the flare and CME of 1996 July 9 from the same active region as the one producing the August 19 type II burst has been shown (Laitinen et al. 2000) to be related with a broadband radio burst that signalled electron acceleration in the low and middle corona independent of a type II shock which was also present during the event (Mann et al. 1999).

We note that the upper limits discussed in this paper were not derived for a weak shock. At a speed near $2000 \mathrm{~km} \mathrm{~s}^{-1}$, the type II shock of 1996 August 19 is one of the fastest large-scale disturbances reported in the solar corona (St. Cyr et al. 1999; Klassen et al. 2000, and references therein). The upper limits hence reflect the restricted ability of an extended coronal shock to accelerate an appropriate number of electrons to high energies. This inefficiency does of course not exclude shock acceleration as such in the low corona, e.g. at fast shocks generated by magnetic reconnection (Aurass et al. 2002; Aschwanden 2002).

Acknowledgements. This work benefitted from the data of EIT/SoHO and the radio experiments aboard Wind and Ulysses through the respective web-pages, as well as from the LASCO/SoHO catalogues of CMEs at Goddard Space Flight Center and the Catholic University of America. KLK acknowledges R. Gorgutsa, S. Hoang, Z. Mouradian, J.C. Noens, M. Reiner and R. Schwenn for providing data from the IZMIRAN radio spectrograph, URAP/Ulysses, Meudon and Pic du Midi $\mathrm{H} \alpha$ instruments, and LASCO/SoHO. He thanks H. Aurass, S. Bale and G. Mann for helpful discussions. The Nançay Radio Observatory is funded by the French Ministry of Education, the CNRS and the Région Centre.

\section{Appendix: Hard X-ray formulae}

In the thick target approximation the injection spectrum of electrons producing a power law photon spectrum with index $\gamma$ is also a power law. The flux of electrons above an assumed lowenergy cutoff $E_{0}$ is (cgs units, adapted from Hudson et al. 1978, Eq. (5))

$$
\begin{aligned}
& \frac{1}{\Phi(50 \mathrm{keV})} \frac{\mathrm{d} N\left(E>E_{0}\right)}{\mathrm{d} t} \\
& \quad=1.64 \times 10^{33}(\gamma-1)^{2} B\left(\gamma-\frac{1}{2}, \frac{1}{2}\right)\left(\frac{E_{0}}{50 \mathrm{keV}}\right)^{-\gamma} .
\end{aligned}
$$

$\Phi(50 \mathrm{keV})$ is the photon flux at $50 \mathrm{keV}, B$ the beta function. The energy flux contained in this population is

$$
\begin{aligned}
\frac{\mathrm{d} W\left(E>E_{0}\right)}{\mathrm{d} t}=8.01 & \times 10^{-8} \frac{\gamma}{\gamma-1} \\
& \times \frac{E_{0}}{50 \mathrm{keV}} \frac{\mathrm{d} N\left(E>E_{0}\right)}{\mathrm{d} t}
\end{aligned}
$$

These equations are used in Sect. 3.2 (Table 2) to compute the flux and energy flux of electrons producing the hard X-ray emission observed by Ulysses, given the parameters of the photon spectrum in Table 1.

The thin target approximation provides the instantaneous number spectrum of X-ray emitting electrons in a plasma with ambient density $n_{0}$. The number of electrons above $E_{0}$ is (after Hudson et al. 1978, Eq. (8))

$$
\begin{aligned}
& \frac{N\left(E>E_{0}\right) n_{0}}{\Phi(50 \mathrm{keV})}= \\
& \quad=3.71 \times 10^{44} \frac{\gamma-1}{\left(\gamma-\frac{3}{2}\right) B\left(\gamma-1, \frac{1}{2}\right)}\left(\frac{E_{0}}{50 \mathrm{keV}}\right)^{3 / 2-\gamma} .
\end{aligned}
$$


These electrons carry the energy (in ergs)

$W\left(E>E_{0}\right)=8.01 \times 10^{-8} \frac{\gamma-\frac{3}{2}}{\gamma-\frac{5}{2}} \frac{E_{0}}{50 \mathrm{keV}} N\left(E>E_{0}\right)$.

Inserting the maximum photon fluxes consistent with the nondetection of X-ray emission by BATSE and GOES (Table 1), we compute (Table 2) the maximum instantaneous number and energy content of electrons in the type II radio source for different assumed photon spectral indices $\gamma$ and low energy cutoffs $E_{0}$.

\section{References}

Aschwanden, M. J. 2002, Space Sci. Rev., 101, 1

Aurass, H. 1997, in Coronal Physics from Radio and Space Observations, ed. G. Trottet (Springer), LNP, 483, 135-160

Aurass, H., Vršnak, B., \& Mann, G. 2002, A\&A, 384, 273

Bale, S. D., Reiner, M. J., Bougeret, J.-L., et al. 1999, Geophys. Res. Lett., 26, 1573

Benz, A. O. 1993, Plasma astrophysics: Kinetic processes in solar and stellar coronae, Astrophysics and Space Science Library, 184 (Dordrecht: Kluwer)

Benz, A. O. \& Thejappa, G. 1988, A\&A, 202, 267

Biesecker, D. A., Thompson, B. J., Gibson, S. E., et al. 1999, JGR, 104, 9679

Bougeret, J. -L., Kaiser, M. L., Kellogg, P. J., et al. 1995, Space Sci. Rev., 71, 231

Cairns, I. H. \& Robinson, R. D. 1987, Sol. Phys., 111, 365

Cane, H. V., Erickson, W. C., \& Prestage, N. P. 2002, JGR, 107, 1315

Cane, H. V., Stone, R. G., Fainberg, J., et al. 1981, Geophys. Res. Letters, 8,1285

Cliver, E. W., Dennis, B. R., Kiplinger, A. L., et al. 1986, ApJ, 305, 920

Crosby, N. B., Aschwanden, M. J., \& Dennis, B. R. 1993, Sol. Phys., 143,275

Démoulin, P., Mandrini, C., van Driel-Gesztelyi, L., et al. 2002, A\&A, 382,650

Dulk, G. A., Leblanc, Y., Bastian, T. S., \& Bougeret, J. L. 2000, JGR, 105,27343

Dulk, G. A., Leblanc, Y., Robinson, P. A., Bougeret, J. L., \& Lin, R. P. 1998, JGR, 103, 17223

Duncan, R. A. 1979, Sol. Phys., 63, 389

Fishman, G. J., Meegan, C. A., Wilson, R. B., et al. 1989, in Gamma Ray Observatory Science Workshop, ed. W. N. Johnson, 2.39

Frost, K. J. \& Dennis, B. R. 1971, ApJ, 165, 655

Garcia, H. A. 1994, Sol. Phys., 154, 275

Holman, G. D. \& Pesses, M. E. 1983, ApJ, 267, 837

Hudson, H. S., Canfield, R. C., \& Kane, S. R. 1978, Sol. Phys., 60, 137

Hudson, H. S., Kosugi, T., Nitta, N. V., \& Shimojo, M. 2001, ApJ, $561, \mathrm{~L} 211$
Hurley, K., Sommer, M., Atteia, J.-L., et al. 1992, A\&AS, 92, 401 Jackson, B. V. \& Leblanc, Y. 1991, Sol. Phys., 136, 361

Kahler, S. W. 1984, Sol. Phys., 90, 133

Kahler, S. W. \& Kreplin, R. W. 1991, Solar Phys., 133, 371

Kane, S. R., Hurley, K., McTiernan, J. M., et al. 1995, ApJ, 446, L47

Kane, S. R., McTiernan, J., Loran, J., et al. 1992, ApJ, 390, 687

Kerdraon, A. \& Delouis, J. 1997, in Coronal Physics from Radio and Space Observations, ed. G. Trottet (Springer), LNP, 483, 192-201

Klassen, A., Aurass, H., Mann, G., \& Thompson, B. J. 2000, A\&AS, 141,357

Klassen, A., Bothmer, V., Mann, G., et al. 2002, A\&A, 385, 1078

Klein, K.-L., Klassen, A., Aurass, H., \& LASCO Consortium 1997, in Fifth SOHO Workshop: The Corona and Solar Wind Near Minimum Activity, ESA SP, 444, 461

Klein, K.-L., Trottet, G., Benz, A. O., \& Kane, S. R. 1988, in Plasma Astrophysics, ESA SP 285 (I), 157

Klein, L., Anderson, K., Pick, M., et al. 1983, Sol. Phys., 84, 295

Koutchmy, S. 1994, in Adv. Space Res., 14(4), 29

Laitinen, T., Klein, K. L., Kocharov, L., et al. 2000, A\&A, 360, 729

Lecacheux, A. 2000, in AGU Monograph, 119, Radio Astronomy at Long Wavelengths, ed. R. Stone, K. Weiler, M. Goldstein, \& J.-L. Bougeret, 321

Lin, R. P. 1985, Sol. Phys., 100, 537

Lin, R. P., Mewaldt, R. A., \& van Hollebeke, M. A. I. 1982, ApJ, 253, 949

Lin, R. P., Schwartz, R. A., Kane, S. R., Pelling, R. M., \& Hurley, K. C. 1984, ApJ, 283, 421

Mann, G. 1995, in Coronal Magnetic Energy Releases, ed. A. O. Benz, \& A. Krüger (Springer), LNP, 444, 183

Mann, G., Aurass, H., Voigt, W., \& Paschke, J. 1992, in Coronal Streamers, Coronal Loops, and Coronal and Solar Wind Composition, ESA SP, 348, 129

Mann, G. \& Classen, H.-T. 1995, A\&A, 304, 576

Mann, G., Classen, H. T., Motschmann, U., Kunow, H., \& Dröge, W. 1999, Ap\&SS, 264, 489

Mann, G. \& Klassen, A. 2002, in Solar Variability: From Core to Outer Frontiers, ESA SP, 506, 245

Nelson, G. J. \& Melrose, D. B. 1985, in Solar Radiophysics: Studies of Emission from the Sun at Metre Wavelengths, ed. D. McLean \& N. Labrum (Cambridge University Press), 333

Simnett, G. M., Roelof, E. C., \& Haggerty, D. K. 2002, ApJ, 579, 854

Slottje, C. 1981, Atlas of fine structures of dynamic spectra of solar type IV-dm and some type II radio bursts (Publ. Utrecht Observatory)

St. Cyr, O. C., Burkepile, J. T., Hundhausen, A. J., \& Lecinski, A. R. 1999, JGR, 104, 12493

Stewart, R. T. 1984, Sol. Phys., 94, 379

Stone, R. G., Bougeret, J. L., Caldwell, J., et al. 1992, A\&AS, 92, 291

Thomas, R. J., Crannell, C. J., \& Starr, R. 1985, Sol. Phys., 95, 323

Zlotnik, E. Y., Klassen, A., Klein, K.-L., Aurass, H., \& Mann, G. 1998, A\&A, 331, 1087 